\title{
Biobanking of Dehydrated Human Donor Corneal Stroma to Increase the Supply of Anterior Lamellar Grafts
}

\author{
Vito Romano, MD, ${ }^{*} \dagger \ddagger$ Hannah J. Levis, PhD,† Paola Gallon, MSc,§ Rebecca Lace, PhD, $\dagger$ \\ Davide Borroni, MD, ${ }^{*}$ Diego Ponzin, MD,§ Alessandro Ruzza, BSc,§ Stephen B. Kaye, \\ $\mathrm{MD},{ }^{*}+$ Stefano Ferrari, PhD, $\S$ and Mohit Parekh, PhD§
}

Purpose: To investigate the effect of dehydration on human donor corneal stroma for biobanking.

\begin{abstract}
Methods: Epithelium and endothelium of research-grade human donor corneas $(n=12)$ were scraped off, leaving a bare stroma with attached sclera. The tissues were placed in a large Petri dish prefilled with silica gel in the periphery and stored at room temperature for 14 days. At the end of preservation, the tissues were rehydrated by being submerged in phosphate-buffered saline for 15 minutes. Transparency (using a custom-built device) and thickness (using optical coherence tomography) measurements were recorded before dehydration, after dehydration, and after rehydration of the tissues. Periodic acid-Schiff and alpha-smooth muscle actin (a-SMA) staining before dehydration and after rehydration were performed to determine the presence of keratocytes and expres- sion of a-SMA. Tensile stress-strain before dehydration and after rehydration was performed to evaluate the biomechanical properties.
\end{abstract}

Results: No difference in corneal transparency before dehydration (69.57 6 6.41\%) and after rehydration (67.37 6 $2.82 \%), \mathrm{P}=0.36$, was observed. The corneas were more compact after dehydration. A significant change in thickness between before dehydration (625.8 $675.58 \mathrm{~mm}$ ) and after rehydration $(563.6615 .77 \mathrm{~mm})$ stage, $\mathrm{P}=0.03$, was noticed. The thickness was reduced to $147.663 .71 \mathrm{~mm}$ when dehydrated. Periodic acid-Schiff staining showed presence of stromal keratocytes and a-SMA protein expressed in control, dehydrated, and rehydrated corneas. There was no significant difference in the stiffness between control (27.86 $611.65 \mathrm{MPa})$ and rehydrated corneas (31.46 6 11.41 MPa).

Conclusions: Human donor corneal stroma can be biobanked for up to 2 weeks in a dehydrated condition without losing their molecular or biomechanical properties after rehydration.

Key Words: cornea, stroma, biobanking, dehydration

\section{Introduction}

Corneal opacity has been reported as the third leading cause of blindness in low- and middle-income countries. ${ }^{1}$ The development of increasingly innovative surgical tech- niques is leading to innumerable advantages, including the possibility of using different layers of a single cornea for different transplantation purposes, as reported in several studies, ${ }^{2-4}$ allowing more patients to be treated. However, the timely availability of donor corneas is not yet sufficient. Therefore, tissue bioengineering is required to provide solutions toward developing a biomimetic, cytocompatible, and transplantable stromal replacement. ${ }^{5}$ According to the European Eye Bank Association directives, tissues with ,2000 to 2200 cells $/ \mathrm{mm}^{2}$ are typically not used for transplantation. However, in certain cases, the stroma from these discarded tissues can be used for other surgeries. Therefore, finding a storage method suitable for the pres- ervation of corneal stroma could be advantageous. Differ- ent dehydration storage conditions have been evaluated by several research groups that include organ culture medium supplemented with 5\% PEG 35,000 for the dehydration of anterior donor grafts, ${ }^{6}$ anhydrous glycerol with and without silica gel, and various temperatures for the preservation of corneal tissues. ${ }^{7}$

To increase the availability of these tissues, we therefore investigated if human donor corneal stroma stored in a dehydrated condition and rehydrated after 2 weeks retained the same biological and biomechanical properties. We used silica gel at room temperature as a dehydrating agent, conditions easily repeatable even in developing countries. Our aim is to have a supply that meets the demand, by biobanking the stromal tissue ready-to-be-used when needed, thereby reducing the amount of discarded corneal tissue. 


\section{MATERIALS AND METHODS}

\section{Ethical Statement}

The corneas ( $\mathrm{n}=12$ ) were obtained from the Veneto Eye Bank Foundation (FBOV, Venice, Italy) with written consent from the donor's next of kin to be used for research. The tissues with a fair number of endothelial cells (1200- 1600 cells $/ \mathrm{mm}^{2}$ ) and with a clear stroma without any noted aberrations were included in this study.

\section{Dehydration and Rehydration Method}

The corneas were placed on a white silicone base. With the endothelium facing the air, the Descemetendothelial complex was stripped using a standard strip- ping method. The tissues were inverted upside down with epithelium facing up. The epithelium was removed using a sterile sponge by a scraping method. All the corneas without epithelium and endothelium were placed in a clock-wise fashion in a large Petri dish with silica gel and stored at room temperature for up to 14 days (see Supplemental Figure 1A, Supplemental Digital Content 1, http://links.lww.com/ICO/A743). All the tissues were dehy- drated simultaneously (see Supplemental Figure 1B, Supple- mental Digital Content 1, http://links.lww.com/ICO/A743) and were analyzed after rehydration by submerging in sterile

phosphate-buffered saline for 15 minutes.

Thickness and Transparency Measurements $(\mathrm{n}=12)$

Thickness of the corneas was monitored using Optical Coherence Tomography (OCT, Tomey, Japan) after mount- ing the corneas on an artificial anterior chamber. Trans- parency was measured using a custombuilt device by checking the light intensity using a probe as described previously. ${ }^{8,9}$ The measurements were obtained 1) after the removal of epithelium and endothelium, 2) after dehydration, and 3) after rehydration of the tissues.

\section{Histology Using PAS Staining $(n=4)$ and Expression of Alpha-Smooth Muscle Actin (a-SMA) Protein $(n=4)$}

The tissues (dehydrated, rehydrated, and controls) were fixed in $4 \%$ paraformaldehyde for 24 hours. The tissues were washed and embedded in optimal cutting temperature (OCT) compound before microtome cutting. The sections were stained using standard PAS protocol. The other sections were washed after paraformaldehyde and permeabilized with Triton X-100 for 30 minutes, washed thrice in phosphatebuffered saline and blocked with $20 \%$ goat serum. The sections were treated with a primary antibody (anti-aSMA; 1:500, ab5694, Cambridge, MA) and incubated at $4^{\circ} \mathrm{C}$ for 24 hours. After washing, a secondary antibody conjugated with FITC was added for 3 hours at room temperature followed by washing. The sections were mounted with DAPI and examined using a Nikon Eclipse Ti-E (Nikon, Amsterdam, The Netherlands) microscope with NIS Elements software (Nikon).

Stiffness $(n=4)$

To investigate if there was a change in stiffness after the dehydration process, 2 control corneas and 4 rehydrated corneas were examined using the Linkam TST bench-top tensile tester (Linkam Scientific, Epsom, United Kingdom) with a $20 \mathrm{~N}$ load cell. All corneas were cut to a width of $2 \mathrm{~mm}$ and the thickness was measured before testing, samples were tested until failure and the stiffness was measured from the stress/strain curve.

\section{Statistical Analysis}

The data are expressed as Mean 6 SD. Statistical analysis was performed using Microsoft Excel for obtaining the mean, SD, and P value, where P , 0.05 was deemed significant. Analysis of variance for paired data was also performed. 


\section{RESULTS}

Thickness

Average thickness values found using OCT (Fig. 1) was 625.8 (675.58) mm before dehydration. Average thickness

after dehydration reduced to 147.6 (63.71) $\mathrm{mm}$ (Fig. 1B). Average thickness observed after rehydration was 563.6 (615.77) $\mathrm{mm}$ (Fig. 1C). Thickness value before dehydration and after rehydration was found to be statistically signifi- cantly different $(\mathrm{P}=0.03)$ (Fig. 1D).

\section{Transparency}

Average transparency before dehydration (Fig. 2A) was found to be 69.57 (66.41)\%. After dehydration (Fig. 2B), average transparency reduced to 52.75 (67.07)\%. After rehydration (Fig. 2C), 67.37 (62.82)\% transparency was observed. Transparency between before-dehydration and after-rehydration did not show any statistical significance $(\mathrm{P}=0.36)($ Fig. 2D).

PAS Staining and Expression of a-SMA Keratocytes were observed in control (Fig. 3A), dehydrated (Fig. 3B), and rehydrated corneal samples (Fig. 3C). a-SMA was expressed in control (Fig. 4A), dehydrated (Fig. 4B), and rehydrated corneas (Fig. 4C), indicating the presence of a-SMA protein in the stroma after rehydration.

\section{Mechanical Properties}

The tissues were not brittle and showed flexibility when folded (Fig. 5A). Average stiffness values found using tensile testing were 27.86 (611.65) MPa for control corneas and 31.46 (611.41) MPa for rehydrated corneas, which was not statistically significantly different $(P=0.72)$ (Fig. 5B). This indicates that the dehydration process did not affect the mechanical properties of the corneal stroma.

\section{DISCUSSION}

Increasing numbers of patients are being treated with new and advanced surgical methods leading to increased demand for already scarcely available tissues. ${ }^{4}$ To overcome this issue, it is proposed that multiple keratoplasties be performed using a single donor cornea increasing the availability of transplantable tissues. ${ }^{4}$ Prolonging the life of these tissues will be key to the success of this approach.

In transplants that involve a portion of stroma, donor stromal cells are replaced with host cells after transplant, suggesting that the donor corneal stroma may act merely as a scaffold. The donor stroma is repopulated with recipient cells by centripetal movement over time although the time for complete replacement depends on multiple factors. ${ }^{10}$ Conse- quently, nonviable (formalinized, lyophilized, and dehy- drated) tissues have been used for anterior keratoplasty because the donor stroma acts merely as a scaffold..$^{10}$ Other studies have shown that freeze-dried tissues can also be used for conditions like epikeratophakia. ${ }^{11,12}$ Tripathi et $\mathrm{al}^{7}$ pro- posed preserving tissues at $280^{\circ} \mathrm{C}$, which maintained the sterility, thickness, optical transparency, mechanical strength, and ultrastructural features. However, there is also contradic- tory evidence suggesting that viable donor keratocytes are necessary for a successful graft until the donor graft is completely attached to the recipient cornea. ${ }^{13}$ Russo et $\mathrm{al}^{14}$ investigated the use of dehydrated stromal lamellae, studying the ability of the host keratocytes to repopulate transplanted lamellae that did not contain viable cells. The group concluded that the repopulation of host keratocytes does not always occur because it requires extensive contact between donor and host parenchyma.

We studied the effect of silica gel dehydration and rehydration on the properties of stromal tissues with histology after PAS staining. Our results show that the stroma maintains the same biomechanical properties after rehydration com- pared with the control group. This suggests that it would be possible to 
biobank the stromal tissues in a dehydrated condition for up to 2 weeks and still retain its structural properties.

In conclusion, tissues discarded because of low endothe- lial cell count or tissue remaining (anterior lamellae) after surgeries such as Descemet Membrane Endothelial Keratoplasty could be kept in dehydrated conditions and used for deep anterior lamellar surgery or for tectonic purposes. This strategy will improve the number of suitable corneas available for transplantation. Because the thickness, transparency, and bio- mechanical properties were not altered significantly by the dehydration and rehydration processes, the stromal tissues could be biobanked in a dehydrated form and used after rehydration once its clinical efficacy has been confirmed. Further studies are needed to explore the possibility of extending the preservation period of dehydrated corneas.

\section{REFERENCES}

1. Feilmeier MR, Tabin GC, Williams L, et al. The use of glycerol- preserved corneas in the developing world. Middle East Afr J Ophthalmol. 2010;17:38-43.

2. Nikolic L, Jovanovic V, Jankov MR II. One cornea for two patients: case report. Arq Bras Oftalmol. 2010;73:291-293.

3. Sharma N, Agarwal P, Titiyal JS, et al. Optimal use of donor corneal tissue: one cornea for two recipients. Cornea. 2011;30:11401144.

4. Vajpayee RB, Sharma N, Jhanji V, et al. One donor cornea for 3 recipients: a new concept for corneal transplantation surgery. Arch Ophthalmol. 2007;125:552-554.

5. MatthyssenS,VandenBogerdB,DhubhghaillSN,etal.Cornealregeneration: a review of stromal replacements. Acta Biomater. 2018;69:31-41.

6. Lie JT, Monnereau C, Groeneveld-van Beek EA, et al. Dehydration of corneal anterior donor tissue with polyethylene glycol (PEG)enriched media. Cell Tissue Bank. 2015;16:399-409.

7. Tripathi H, Mehdi MU, Gupta D, et al. Long-term preservation of donor corneas in glycerol for keratoplasty: exploring new protocols. Br J Ophthalmol. 2016;100:284-290.

8. Parekh M, Salvalaio G, Ferrari S, et al. A quantitative method to evaluate the donor corneal tissue quality used in a comparative study between two hypothermic preservation media. Cell Tissue Bank. 2014;15:543-554.

9. Parekh M, Ferrari S, Ruzza A, et al. A portable device for measuring donor corneal transparency in eye banks. Cell Tissue Bank. 2014;15:7-13.

10. Wollensak G, Green WR. Analysis of sex-mismatched human corneal transplants by fluorescence in situ hybridization of the sexchromosomes. Exp Eye Res. 1999;68:341e6.

11. Kaufman HE, Werblin TP. Epikeratophakia for the treatment of keratoconus. Am J Ophthalmol. 1982;93:342-347.

12. Rostron CK, Sandford-Smith JH, Morton DB. Experimental epikerato- phakia using tissue lathed at room temperature. Br J Ophthalmol. 1988; 72:354-360.

13. Maumenee AE, Komblueth W. Corneal transplantation; physiopathol- ogy. Am J Ophthalmol. 1948;31:1384e93.

14. Russo A, Bonci P, Leonetti $P$, et al. Long-term dehydrated donor lamella survival in anterior keratoplasty: keratocyte migration and repopulation of corneal stroma. Cornea. 2015;34:1044-1051. 

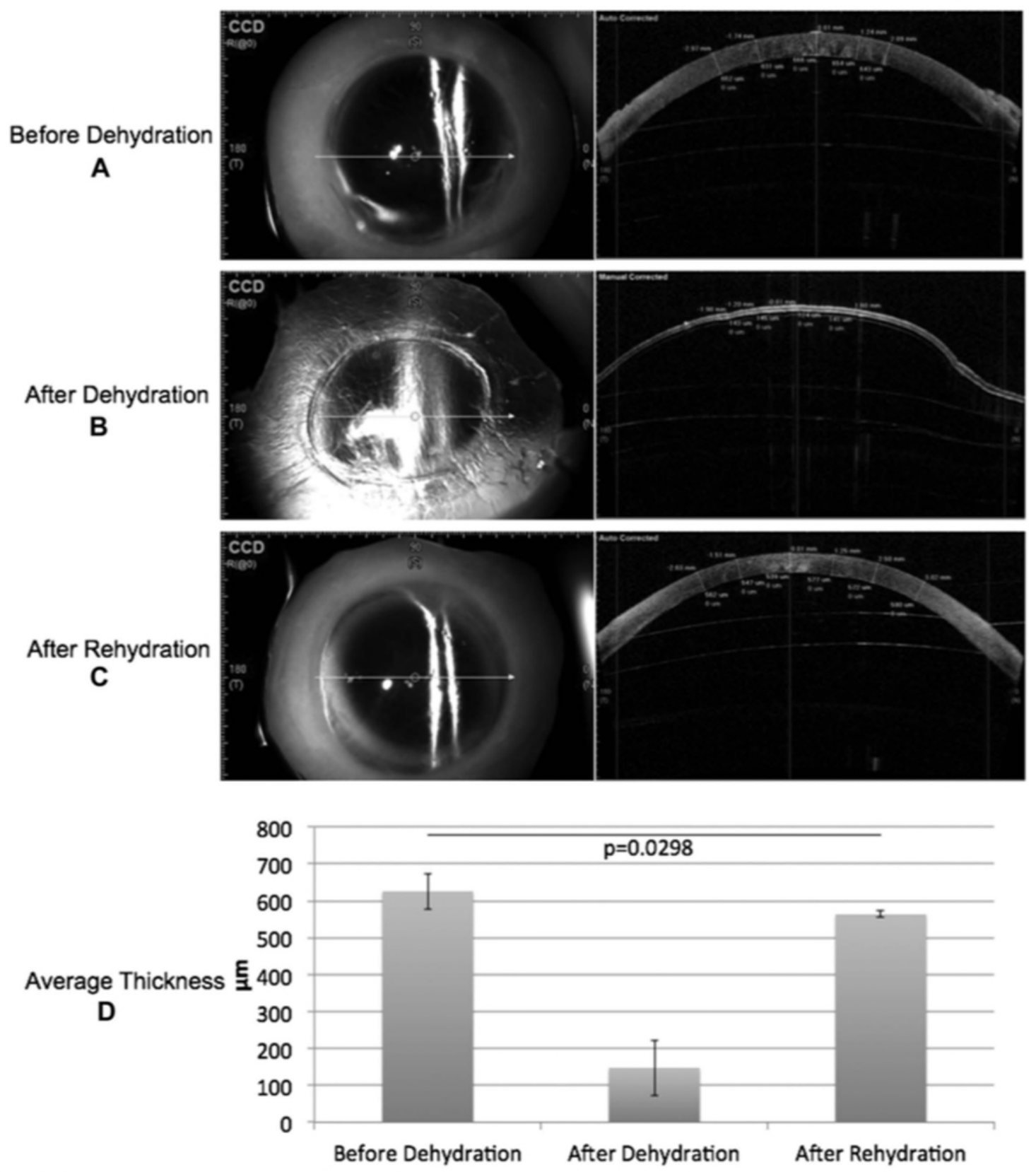

FIGURE 1. Thickness measurements using optical coherence tomography (A) before dehydration, (B) after dehydration, and (C) after rehydration. D, Thickness was found to be significantly different before dehydration and after rehydration. 


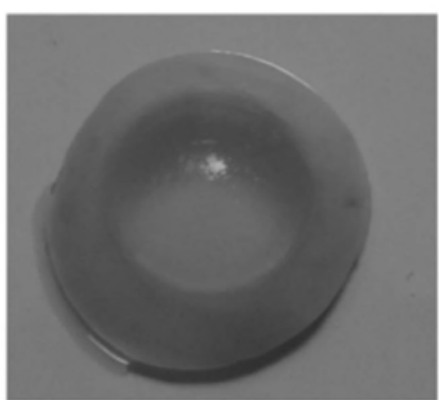

A

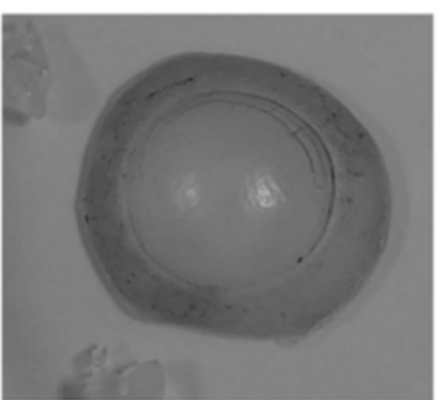

B

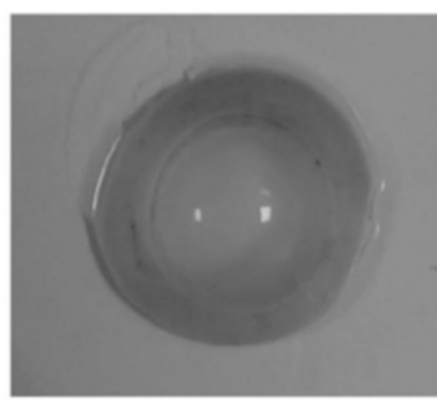

C

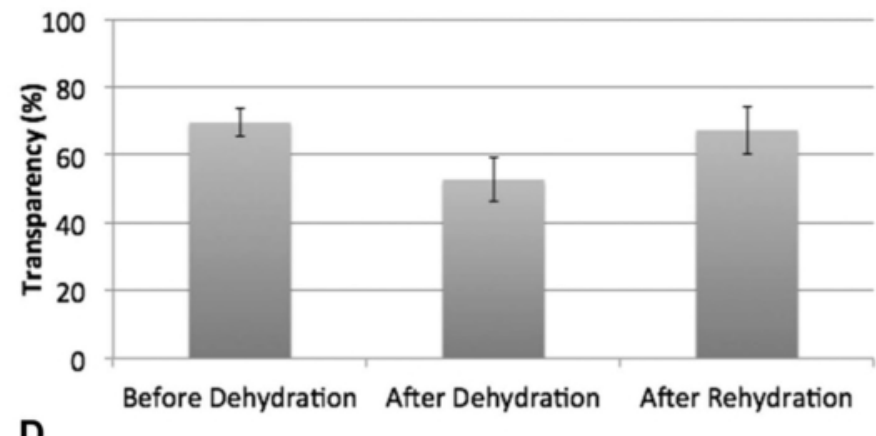

FIGURE 2. Transparency of the corneas (A) before dehydration, (B) after dehydration, and (C) after rehydration. D, No significant difference was found in transparency readings using a custom-built device.

FIGURE 3. Periodic acid-Schiff (PAS) staining of the corneas at $\times 200$ magnification in (A) control corneas (untreated samples), (B) dehydrated corneas, and $(C)$ corneas after rehydration. Arrow marks show the pres ence of keratocytes in each condition.
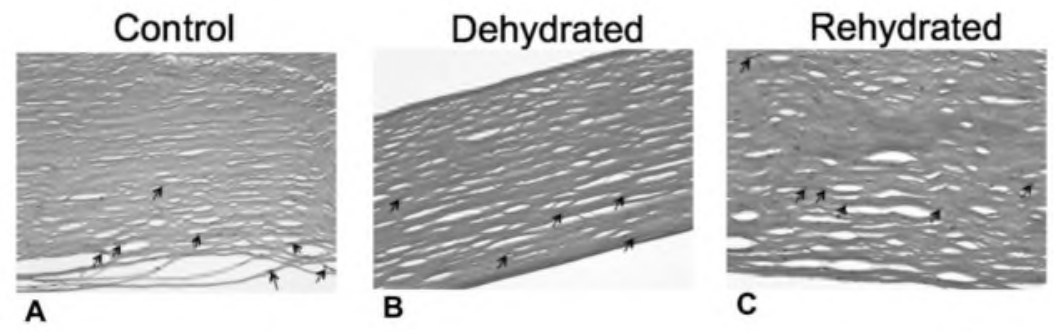

C

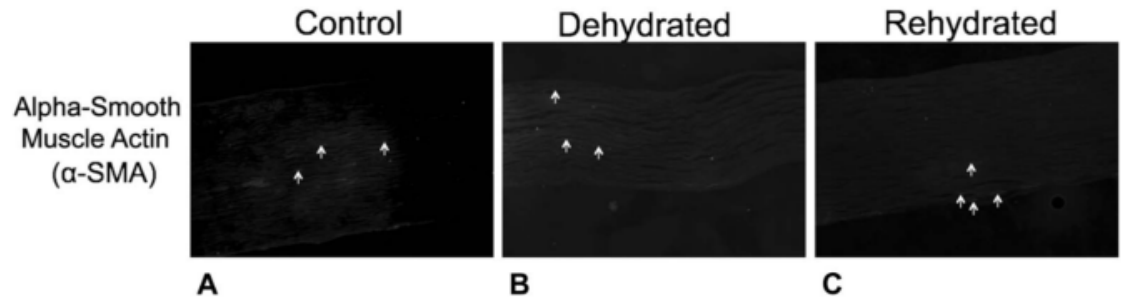

FIGURE 4. Alpha-smooth muscle actin ( $\alpha$-SMA) was expressed in all the conditions; (A) control, (B) dehydrated, and (C) rehydrated corneas showing that dehydration or following rehydration does not affect the expression of $\alpha$-SMA in the stroma. The arrows indicate keratocytes stained with alpha-SMA. 
FIGURE 5. Flexibility and mechanical properties of the rehydrated tissue. A Folding of the tissue showing no brittleness with high flexibility. B, Box plot average stiffness measured in $\mathrm{MPa}$ of control and rehydrated corneas. There was no significant difference in stiffness between control corneas and corneas that had been rehydrated; therefore, the mechanical properties do not seem to be influenced by the dehydration step.
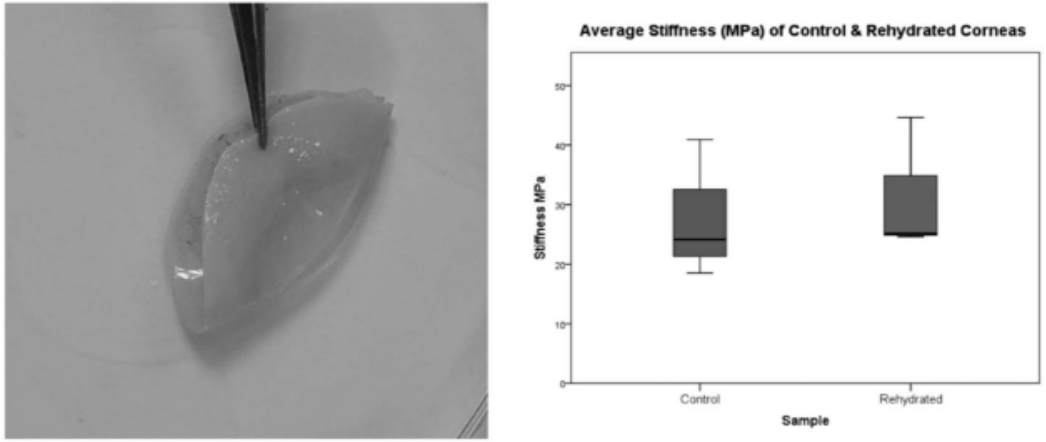

A 